\title{
Evaluation of an Automatically-Constructed Graph-Based Representation for Interactive Narrative
}

\author{
Nathan Partlan \\ nkp@ccs.neu.edu \\ Northeastern University \\ Sam Snodgrass \\ s.snodgrass@northeastern.edu \\ Northeastern University \\ Camillia Matuk \\ cmatuk@nyu.edu \\ New York University
}

\author{
Elin Carstensdottir \\ elin@ccs.neu.edu \\ Northeastern University \\ Casper Harteveld \\ c.harteveld@northeastern.edu \\ Northeastern University \\ Steven C. Sutherland \\ sutherland@uhcl.edu \\ University of Houston-Clear Lake
}

\author{
Erica Kleinman \\ erica@ccs.neu.edu \\ Northeastern University \\ Gillian Smith \\ gmsmith@wpi.edu \\ Worcester Polytechnic Institute \\ Magy Seif El-Nasr \\ m.seifel-nasr@northeastern.edu \\ Northeastern University
}

\begin{abstract}
Interactivity and player experience are inextricably entwined with the creation of compelling narratives for interactive digital media. Narrative shapes and buttresses many such experiences, and therefore designers must construct compelling narrative arcs while carefully considering the effects of interaction on both the story and the player. As the narrative becomes more structurally complex, due to choice-based branching and other player actions, designers need to employ commensurately capable models and visualizations to keep track of that growing complexity. However, previous models of interactive narrative have failed to fully capture interactive elements with automated, operationalized visualizations. In this paper, we describe an algorithm for automated construction of a framework-driven, graph-based representation of interactive narrative. This representation more fully and transparently models structural and interactive features of the narrative than did prior approaches. We present an initial evaluation of this representation, based on modified cognitive walkthroughs performed by interactive narrative design and research experts from our research team, and we describe the takeaways for future improvement on interactive narrative modeling and analysis.
\end{abstract}

\section{CCS CONCEPTS}

- Computing methodologies $\rightarrow$ Knowledge representation and reasoning; Simulation evaluation; • Applied computing $\rightarrow$ Computer games; $\bullet$ Software and its engineering $\rightarrow$ Design languages.

\footnotetext{
Permission to make digital or hard copies of all or part of this work for personal or classroom use is granted without fee provided that copies are not made or distributed for profit or commercial advantage and that copies bear this notice and the full citation on the first page. Copyrights for components of this work owned by others than the author(s) must be honored. Abstracting with credit is permitted. To copy otherwise, or republish, to post on servers or to redistribute to lists, requires prior specific permission and/or a fee. Request permissions from permissions@acm.org.

FDG '19, August 26-30, 2019, San Luis Obispo, CA, USA

(c) 2019 Copyright held by the owner/author(s). Publication rights licensed to ACM. ACM ISBN 978-1-4503-7217-6/19/08 ..\$15.00

https://doi.org/10.1145/3337722.3341858
}

\section{KEYWORDS}

Games, Interactive Narrative, AI, Computational Co-Creativity, Graphical Models, Design Representation, Structural Models, Design, Computational Media, Player Agency, Knowledge Representation

\section{ACM Reference Format:}

Nathan Partlan, Elin Carstensdottir, Erica Kleinman, Sam Snodgrass, Casper Harteveld, Gillian Smith, Camillia Matuk, Steven C. Sutherland, and Magy Seif El-Nasr. 2019. Evaluation of an Automatically-Constructed Graph-Based Representation for Interactive Narrative. In The Fourteenth International Conference on the Foundations of Digital Games (FDG '19), August 26-30, 2019, San Luis Obispo, CA, USA. ACM, New York, NY, USA, 9 pages. https: //doi.org/10.1145/3337722.3341858

\section{INTRODUCTION}

When building any interactive experience, such as a game, it is critical for the designer to deeply consider and shape its interactive elements. They must form models of the player's likely experiences and interactions, and consider how the player's actions can impact their artifact and inform its reactions. This dialogue between the designer's vision and the players' perceptions shapes and defines the artistic and experiential results of the work.

With the increasing complexity of modern games, these experiences may be many and varied; they may depend deeply on the player's choices, and the designer may have difficulty theorizing about player experience in a vacuum. Narrative-heavy games, such as roleplaying games and visual novels, can encompass a broad spectrum of designs. They can range from linear, movie-like experiences to highly open and branching ones. As a result, designers must make careful choices about how to structure their games to achieve their desired player experiences.

Interactive narrative often requires complex and intensive authoring to track and maintain the narrative through many potential choices and interactions. Authoring tools can assist designers in building a coherent, satisfying narrative by representing and visualizing the design appropriately. A good tool can organize important information, reducing the need for designers to keep separate, complex mental or physical models. Branches, choices, and other player interactions are sources of complexity and may benefit from helpful, clear representation. Authoring tools that can automatically assist 
the designer in representing and reflecting on their design could prove valuable in aiding their analysis of these experiential aspects of their work.

Interaction, in this context, encompasses the flow and structure of the narrative, the opportunities for player input, and the responses to that input - including story progression, user interface, feedback, animation, and more [8]. When representing interactive narrative, it is important to capture all of these, and to present them in an approachable and comprehensible manner. This representation can be understood as an analogue to the "knowledge representation" component of an agent-based AI. It provides the knowledge base about the "world" of the game that can be used by other AI systems for creativity support and automated playtesting.

In recent work, we proposed such a representation, composed of multiple graphs to capture the scene flow, layout, scripts, and interactions [34]. However, this prior work did not fully detail the methods for computationally constructing such a representation, nor did it deeply explore the effectiveness of the representation in illuminating the design. It also neglected to deeply interrogate the representation's flaws and limitations. Here, we describe the computational process for building such a representation, as well as an initial expert evaluation that we performed in order to evaluate and find opportunities for improvement in that representation.

The representation is implemented in the StudyCrafter platform for interactive narrative scenario creation. The tool enables novice designers to develop playable scenarios, often for the purpose of performing social science experiments [18]. StudyCrafter has been employed in several classrooms, resulting in a small body of studentbuilt experiments that have been found to represent a variety of styles, strengths, and weaknesses [34]. We use two of those experiments, "An Unusual Situation" and "The Research Riddle," as the subjects of our evaluation.

Our evaluation consists of in-person, in-depth interviews with members of our research team. Each researcher critically examined the representations of individual interactive scenarios using a modified cognitive walkthrough method [25]. This work's primary contribution is to describe and critically evaluate our computational representation of interactive narrative scenarios, and outline requirements for developing a representation that fully encompasses and exposes a scenario's interaction patterns and potential experiences. This understanding enables improvements to computational narrative analysis and future work on AI support for interactive narrative design, with a focus on modeling player interactions in a way that is interpretable and usable for designers.

\section{RELATED WORK}

Academic analysis of interactive narrative has a long history, dating back at least to the early 1980s with Laurel's work based on Neo-Aristotelian theory [22], if not earlier [31, 37]. In these analyses, however, comprehensively representing player interaction has not usually been the primary focus. Most work has explored the potential and best-practices for authorship [27, 32, 40] and defined interactive narrative structure from the designer's perspective, as in the works of Bernstein, Lindley, Ashwell, Short, and others $[2,4,24,30]$. While these structures are useful for tracking the potential branches of a story, they do not explicitly represent the details of the player's interaction with that structure.

In its totality, interaction includes such elements as the structural and choice affordances, narrative progression, UI, and other forms of feedback for player action. These elements were explored and outlined in recent work by Carstensdottir et. al. [8]. More recently, this research has expanded into a proposal for "interaction maps," designed to capture some of these elements of interaction [9]. Here, we measure that framework and explore the ways in which it successfully captures elements of interaction, and, more importantly, the opportunities for improving it.

Player experience in interactive narrative has also been studied separately from structure, as in research on agency $[7,11,15,45,46$, $48]$, and on engagement and immersion [5, 6, 10, 13, 14, 29, 39]. This work, however, has usually operated at the level of holistic measurement of player experience, rather than at the level of detailed representation of each interaction.

In the IRIS project and following work, researchers have begun an effort to more directly extract and separate metrics of player experience in interactive narrative [35, 36, 47]. These metrics are promising, but they do not yet propose a fully computational, operationalized model of interactive narrative that would enable their proposed measurements and support designers. Recently, Partlan et. al. [34] developed such a representation to measure and expand on the "objective metrics" proposed by Szilas and Ilea [44] as part of that project, and here we evaluate and suggest improvements to that representation.

Outside of interactive narrative, researchers have performed formal modeling and analysis of game design. One of the most comprehensive such efforts is the development of Operational Logics, a theory that categorizes many families of game design elements [28]. To operationalize these logics, Osborn [33] has since proposed computational models of specific logics. The algorithm below can be seen as formally representing certain logics from several families, such as character-state, control, and linking logics.

Computational analyses of game design have employed graphs, such as Petri nets [1,23] and Bayes nets [17]. Petri nets, however, quickly become too complex for manual analysis. Our layout graph, described below, is inspired by the D-nodes in Guzdial and Reidl [17], but they focus on physics-based interactions extracted from gameplay videos, for machine learning and game generation, which does not necessarily support manual analysis nor interactive narrative. Bakkes and Dormans [3] proposed modeling mission design and physical level layout as two related graphs, but their work applied specifically to physical level layouts and was primarily generative. They did not deeply explore models of player interaction, design metrics, nor how to interpret existing designs into graphs.

Other researchers have developed programming-language-based models of game design, such as those proposed by Martens and Hammer [26], Gemini by Summerville et. al. [43] and the VGDL by Schaul [38]. These models are still in the early stages, however, and it is unclear how interpretable they will be for game designers. Our work focuses on modeling interactivity, narrative, and gameplay in transparent and visible form, to enable tractable analysis.

Finally, some researchers are exploring automated playtesting, in which AI agents play through games either in their original form or 


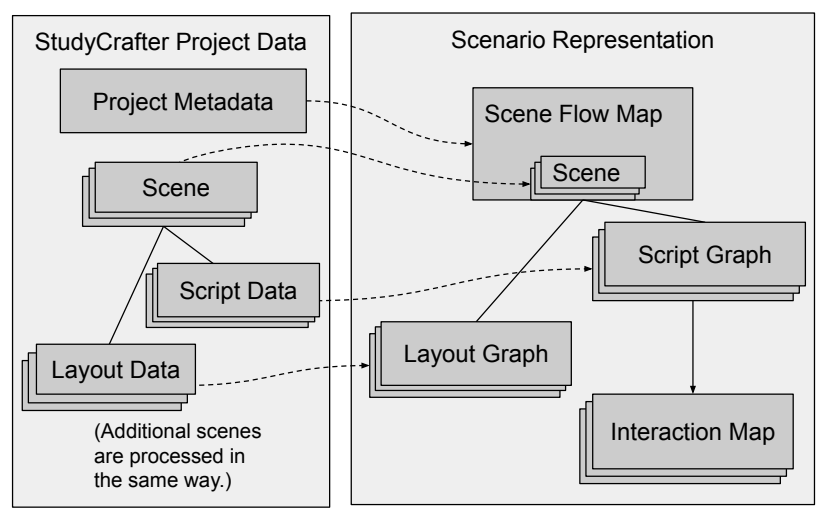

Figure 1: The process for constructing the representation, showing the representation model and relationships between the graphs, as well as the flow of data into the representation.

in simplified simulations [12, 16, 19-21]. Our work can enable automated playtesting by providing a simplified simulation of potential player interactions, obviating the need to fully simulate the physics, animations, and other computationally intensive mechanics of the original game logic. An initial approach to automated playthroughs of the below representation can be found in [34]. Augmenting manual analysis with robust automated playtesting is one exciting area for future exploration that stems from this work.

\section{COMPUTING THE REPRESENTATION}

The automated representation of interactive narrative consists of four types of related graphs: the scene graph, layout graph, script graph, and interaction map. A description of the theory behind these graphs and their use in calculating automated metrics can be found in prior work by Carstensdottir and Seif El Nasr [9] and Partlan et. al. [34]. Below, we describe the algorithm for constructing the representation from a StudyCrafter project, as shown in Figure 1.

\subsection{The Scene Flow Map}

Most StudyCrafter scenarios are choice-based interactive narratives, similar to those that could be developed using other authoring tools such as Twine. In StudyCrafter, the story is broken down into scenes, each of which is a single backdrop for part of the action, much like scenes in theater or visual novels. Each scene in the original scenario contains the visual and scripting data for that scene. For each scene, we create a node in the scene flow map.

The scene flow map represents how the scenes connect to each other. In StudyCrafter, "end" points in the script for each scene contain a property that determines what scene will be loaded next. We use these to build the edges of the scene flow map. Each scene's out-edges lead to the next scenes that may be shown, depending on the player's actions.

For each scene, we build layout and script graphs, and we postprocess the script graph into the interaction map. The scene flow map ties the representation together by keeping references to these other graphs associated with each scene.

\subsection{The Layout Graph}

There is a layout graph for each scene, representing the physical placement of objects in the visual environment. The scene may contain animation, but the layout graph contains information only about the initial placement of the objects. To construct the layout graph, we copy the properties of each object from the saved scene data, including its position, name, type, image, and so forth. We then build edges between each object and each other object, and we annotate the edges with the distances and directions between the objects' centers.

\subsection{The Script Graph}

The script graph for each scene contains the code to operate the scene's gameplay logic. StudyCrafter already contains a visual scripting language, which is displayed with a partially-connected graph-like view in the StudyCrafter editor. Our representation rebuilds this as a true directed graph, ensuring that we can use standard graph algorithms to operate on it.

We keep a data-driven mapping of StudyCrafter script node types to our representation, with specifications of the properties they may contain, including the types of data those properties represent. As we encounter each node in the saved script data, we look up this mapping and load each of its properties into an appropriately typed object. Each script node, in addition to containing a set of properties, has a unique ID that we use to connect it with other script nodes. Specifically, we analyze the potential transitions from each script node, based on its type, and connect it to all nodes to which it may transition next. This process differs for different types of script nodes; for instance, an animation node has a single "next" node, whereas a branch node contains one or more condition statements, each of which may transition to a different node.

\subsection{The Interaction Map}

We post-process the script graph using static graph analysis to build the interaction map. This is possible because we have already built the graph-based representation of the script, enabling us to search through it and create nodes and edges based on its structure.

To build the interaction map, we begin by performing a depthfirst search over the script graph. After building the start node, at each edge between nodes in the script, we construct the proper node type for the end of the edge, if applicable, by inspecting the script node. If the script node has a visible or interactive impact, such as displaying dialogue, animating a character, or modifying the UI, we build a new node of undetermined type; it might be a feedback or event node, depending on as-yet unknown context (i.e., whether it is related to prior choices by the player). If it has no direct impact on the player's perception of the scenario (e.g. a hidden branch or changing a variable value), we build a temporary, "non-interaction" node just for tracking its edges, to be removed in post-processing. If it is a choice or event-based interaction, we build an interaction point vertex. We connect each edge as in the script.

There are a few special cases for the construction process. When we come across script nodes that are not related to player input, such as special events based on the passage of time or based on random branching, we mark them as event nodes, since they are 


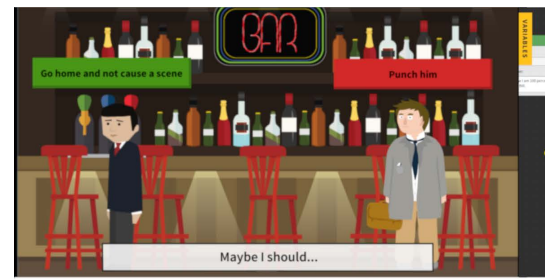

a. Play through the scenario.

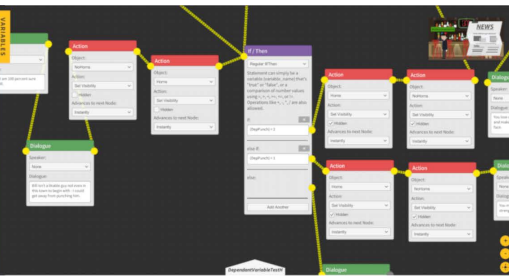

b. Walk through the script.

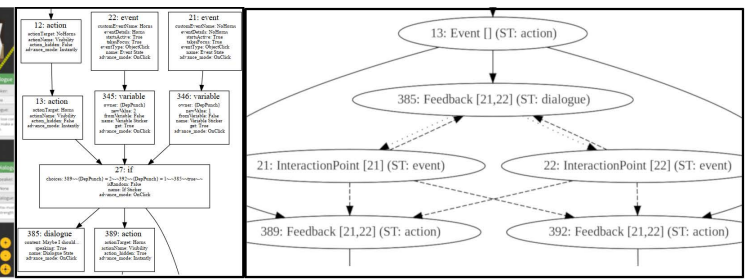

c. Walk through the representation.

Figure 2: The evaluation study process: (a) playing through a scenario, (b) viewing the script in the StudyCrafter visual script editor, and (c) walking through the representation (the script graph is on the left, and the interaction map is on the right).

clearly not feedback. When we find functions, which can be called from multiple points in the script, we connect them as if they directly follow from each of their call points. Finally, when we find end nodes, we mark them with a special end node type.

There are several post-processing steps to complete the creation of the interaction map. We destroy non-interaction nodes and connect their predecessors and successors to each other, simplifying the map. We then need to determine which nodes are related to which interaction units. To do this, we perform a search through the graph starting at each interaction point, where other interaction points are treated as impassible (infinite cost). All reachable nodes, those that have a non-infinite cost, are marked with the interaction unit from which we began the search. We can now mark all nodes of unknown type that are related to an interaction unit as feedback, and all others as events (since they are unrelated to player input).

Finally, we must decide what to do with interaction points that can be triggered by the player at multiple times, such as buttonpress-based events. To let automated playthroughs traverse these events rather than getting stuck, we need to ensure that they have entry edges. In theory, there could be edges to these events from many nodes in the scene, as they can be triggered at multiple times. However, this would clutter the graphs. Therefore, we currently add indirect edges to events only from nodes that would otherwise be dead ends or cycles.

Currently, we build one interaction map for each scene. In theory, we could also build a map of the entire scenario, by combining the scene-based maps. This, however, we leave to future work.

\section{EVALUATION METHOD}

One researcher led three evaluations with different subsets of our research team serving as experts. Each expert was academically trained in computer science and game design, with three of the four experts having experience studying and analyzing games that involve narrative elements and performing player experience research. The fourth had experience with game AI and player modeling research. The experts also had prior experience with StudyCrafter and the representation. In two of the evaluations, separate pairs of researchers evaluated the representation for "An Unusual Situation." In the third evaluation, a single researcher (who also was involved in one of the former evaluations) evaluated the representation for "The Research Riddle."

The evaluation employed a modified cognitive walkthrough method. Cognitive walkthroughs are forms of usability analysis in which the facilitator steps the evaluators through the process of completing a task, asking questions at each step. The original questions are directly tied to usability (i.e. "Will the user notice that the correct action is available?") [25]. We therefore replace the original questions with our own, designed to apply directly to the evaluation of a representation of interactive narrative scenarios. In our method, we perform two walkthroughs: one on the designer's original view of the script of the scenario within the StudyCrafter editor itself, and one on our current graph-based representation.

In the first step in the protocol, experts play through the scenario to be studied using the StudyCrafter scenario player, see Figure 2 (a), as many times as they feel they need to understand and experience it in its entirety. After the experts are comfortable enough with the scenario to understand the script, the first modified cognitive walkthrough begins. StudyCrafter's built-in visual script editor, shown in Figure 2 (b), serves as our point of reference for the existing visual representations for interactive narrative design tools. As experts examine the script for each individual scene in the scenario, we ask the following questions:

(1) What narrative structural features does this scene have that you would want to capture in a representation?

(2) What interaction features does this scene have that you would want to see in the representation?

(3) What other experiential features of this scene do you want to capture in the representation?

These questions are designed to target the narrative structure, the scenario's interactions with the player, and any other aspects of the scenario's design that affect the player's experience. After recording the observations about each of these questions, we move on to the next scene in the scenario.

After completing the walkthrough of the script, Figure 2 (b), we progress to the second modified cognitive walkthrough. This second walkthrough uses the visualizations of the script graphs and interaction maps as described by Partlan et. al. [34] and seen in Figure 2 (c). As the experts examine these representations, we ask the following questions:

(1) Would the representation, assuming it has been modified to capture anything already noted as missing earlier in the walkthrough, give you all the information you need about player interaction and narrative for this scene?

(2) (If the answer is "no" to question 1) What information should be captured that currently is not? 
We expect answers to these questions to be related to the desires for the representation that the experts formulate based on the first walkthrough. We also expect unforeseen problems and new ideas to emerge as the experts examine and react to the existing representation. During this process, for both walkthroughs, new ideas are noted as soon as they are clearly described, after which the team moves on. This is to prevent defensiveness or long design discussions, an approach recommended by Spencer [42].

\subsection{Evaluation Scenarios}

The two scenarios employed in our evaluation process were created by game design students in a graduate-level research methods course. The students were prompted to design scenarios that would serve as social science or psychology experiments.

“An Unusual Situation” begins with a framing narrative and tutorial. Then, it presents a narrative in which the player's character is, depending on an initial randomized condition, either publicly praised or insulted by an old friend in front of a large gathering. Next, the player is given a choice to punch or not punch someone who looks like that friend. Finally, the scenario presents a debrief about the research goals and theories involved.

In "The Research Riddle," the player is either given a specific character or asked to choose between several characters. Then, they are presented with a situation in which a researcher needs their help in finding the answer to a (made up) academic question. To find the answer, the player must navigate a looping set of user interface menus, choices, and text entry fields, simulating a directed search through journals and computer software. ${ }^{1}$

We selected these two scenarios based on their use of interactive narrative elements. They were chosen due to their employment of a variety of interactive affordances, including character selection, UI interactions, dialogue choices, and text entry. Compared to other available scenarios, their narrative framing, characterization, and feedback for player choices presented more opportunities for analysis and discussion.

\subsection{Data Analysis}

We analyzed the results of the cognitive walkthrough-based evaluation by extracting all comments that were relevant to the representation or user experience, and categorizing them into emergent groups based on their themes. We separated comments first by their topic: the experiences of playing through the scenarios, insights available from the original StudyCrafter scripts, experts' expectations for the computational representation, and comments about the actual representation. In each topic, we further defined themes: reflections on narrative design and structure, player interaction, content and characters, and experiment design and style.

\section{RESULTS}

The analysis produced four distinct themes, and further sub-themes within each, derived from the expert comments. Table 1 lists these themes for the playthroughs and script walkthroughs, and Table 2 does so for the representation expectation discussions and walkthrough-based reviews. In this section, we will elaborate on

\footnotetext{
${ }^{1}$ These scenarios are available in the Northeastern University Library archive, at http://hdl.handle.net/2047/D20291320
}

particularly important subsets of the themes. We combine our discussions of the playthrough and script walkthrough sections of Table 1 for brevity, as both sets of comments relate to the original StudyCrafter representation of the scenarios.

\subsection{Narrative Design and Structure}

Comments related to this theme tended to discuss how and to what extent the narrative changed in response to player choices. They also related to the use of particular narrative patterns, such as framing narrative or exposition. For a more complete discussion of structures of interactive narrative, see [8], as well as [2, 4, 24, 41].

Playthrough and Script: During the playthroughs and script walkthroughs, the experts most frequently commented on the linearity of the scenarios. They often focused on structural themes, such as the lack of structural impact of choices and the looping and hidden structures of puzzles.

Representation Expectations: The experts expected the representation to clarify what conditions led to specific branches. If variables were used to create long-term dependencies or branches on prior choices, they wanted the representation to capture that. They also wanted to be able to see randomized variable assignments.

Representation Review: In general, the experts felt that the representation successfully captured linear and branching structures. They felt, however, that it needed more details, especially in terms of dependencies.

\subsection{Player Interaction}

Player interaction encapsulates comments about the types and frequencies of player-driven choices and actions, the feedback for and results of those actions, or the lack thereof.

Playthrough and Script: The experts repeatedly discussed agency. For example, in "The Research Riddle," character selection had too little impact on the story to lead to feelings of agency. They also noted that the provided options were sometimes unexpected or did not reflect all actions the player might want to take.

Representation Expectations: The experts hoped to clearly see how and when the scenario provides feedback for actions, and whether branching is visible or invisible.

Representation Review: The experts agreed that the feedback was clearly marked, and that branching was visible. In general, they had mostly positive comments about this theme. However, they noted a need to account for differing perspectives between players.

\subsection{Content and Characters}

Though the representation focuses on structure and interactivity, the experts often commented on the aesthetic and characterization elements of the narrative.

Playthrough and Script: The experts discussed the relationships between, perspectives of, and setting behind the characters, as well as the player's and narrator's relationships to those elements. In "An Unusual Situation," for example, they pointed out the scenario's use of an unreliable narrator.

Representation Expectations: The experts expressed a desire to see analysis and context for dialogue and narration, including character relationships and perspectives. 


\begin{tabular}{|c|c|}
\hline \multicolumn{2}{|c|}{ An Unusual Situation } \\
\hline Playthrough & Script \\
\hline \multicolumn{2}{|c|}{ Narrative Design and Structure } \\
\hline $\begin{array}{l}\text { Too much framing narrative. } \\
\text { Linear, with a brief foldback. }\end{array}$ & $\begin{array}{l}\text { Choice-driven branches show some differences in length. } \\
\text { Linearity and foldback are visible. } \\
\text { Hidden branches are not visible on first playthrough. }\end{array}$ \\
\hline \multicolumn{2}{|c|}{ Player Interaction } \\
\hline $\begin{array}{l}\text { Felt like it used a "cheap trick": used intentional deception and an } \\
\text { unreliable narrator. } \\
\text { Options did not reflect all actions the player might want to take. } \\
\text { The main character sometimes acted without player input. } \\
\text { Lack of choices and gameplay. } \\
\text { Tutorial introduces simple button interaction. } \\
\text { Provided an unexpected choice. }\end{array}$ & $\begin{array}{l}\text { Can differentiate types of feedback and choices (animation vs. } \\
\text { dialogue, forced vs. open choices, etc.) } \\
\text { "Click to continue" is not a meaningful interaction. } \\
\text { Some "choices" don't matter. }\end{array}$ \\
\hline \multicolumn{2}{|c|}{ Content and Characters } \\
\hline $\begin{array}{l}\text { A phone becomes a character. } \\
\text { Used an unreliable narrator. }\end{array}$ & $\begin{array}{l}\text { Can tell which character is speaking, or if narrator is. } \\
\text { Can see use of props. }\end{array}$ \\
\hline \multicolumn{2}{|c|}{ Experiment Design and Style } \\
\hline $\begin{array}{l}\text { Primary experimental choice lacks context and emotional impact. } \\
\text { Experimental design is not sufficient to test the hypothesis. }\end{array}$ & $\begin{array}{l}\text { Messy, disorganized script layout. } \\
\text { Unusual, button + event-based method of creating a choice. } \\
\text { Scene change for dramatic effect. }\end{array}$ \\
\hline \multicolumn{2}{|c|}{ The Research Riddle } \\
\hline Playthrough & Script \\
\hline \multicolumn{2}{|c|}{ Narrative Design and Structure } \\
\hline $\begin{array}{l}\text { Character selection had no meaningful impact. } \\
\text { Linear with Hidden structures. }\end{array}$ & $\begin{array}{l}\text { Script branches based on prior choices. } \\
\text { Hidden structure is seen in looping script. } \\
\text { Hidden branches are not visible on first playthrough. }\end{array}$ \\
\hline \multicolumn{2}{|c|}{ Player Interaction } \\
\hline $\begin{array}{l}\text { Lack of choices, UI-heavy gameplay. } \\
\text { Interactions felt linear, despite looping, because of single solution. }\end{array}$ & $\begin{array}{l}\text { Not much feedback for character selection. } \\
\text { Can differentiate context of interactions, i.e. "character selection." }\end{array}$ \\
\hline \multicolumn{2}{|c|}{ Content and Characters } \\
\hline $\begin{array}{l}\text { Lack of diversity in characters. } \\
\text { Player was not shown their avatar while solving puzzle. }\end{array}$ & $\begin{array}{l}\text { Can tell which character is speaking, or if narrator is. } \\
\text { Some content changes based on character choice, but does not } \\
\text { affect path of narrative. }\end{array}$ \\
\hline \multicolumn{2}{|c|}{ Experiment Design and Style } \\
\hline Scenario felt detached from the characterization. & Used looping, puzzle-like style, but still linear (one solution). \\
\hline
\end{tabular}

Table 1: The playthrough and script walkthrough themes, organized by category.

Representation Review: The representation did not fulfill most of these expectations, since it is heavily focused on structure. One easily addressable comment was that the "caption" presented alongside certain dialogue choices should be treated separately, as either an event or feedback.

\subsection{Experiment Design and Style}

This theme reflects the experts' comments on how the scenario design and the designer's individual stylistic choices led to particular experiences and affected the experimental validity.

Playthrough and Script: "An Unusual Situation" presents an example of this interplay between stylistic choices and player experience. Experts felt that the choices and unreliable narrator were artificial and manipulative. As a result, they argued that it was unsuccessful in testing its hypothesis.
Representation Expectations: The experts sought clarity on the types and presentation styles of choices, interactions, and feedback. They also wanted information about the context of the scenario and its relationship with the player.

Representation Review: The experts found the representation confusing in some cases, due to designers' stylistic preferences causing unusual results. They also recommended collapsing and combining nodes to reduce complexity.

\section{DISCUSSION}

How do we make sense of these detailed and complex insights gleaned from the cognitive walkthrough process? We begin by determining which aspects of the representation were successful, according to our experts' goals, and then continue by analyzing the desires, concerns, and comments about the representation to find actionable, operationalizable feedback. 


\begin{tabular}{|c|c|}
\hline Representation Expectations & Representation Review \\
\hline \multicolumn{2}{|c|}{ Narrative Design and Structure } \\
\hline $\begin{array}{l}\text { Capture the structural consequences of choices. } \\
\text { Capture the between-scene impacts of decisions. } \\
\text { Show the ordering constraints between interactions. } \\
\text { Show use of variables: branching and long-term dependencies, } \\
\text { visibility in interface, randomized assignment. }\end{array}$ & $\begin{array}{l}\text { (+) Successfully captures structure (linearity, foldback, hidden, etc.) } \\
\text { (-) Difficult to determine dependencies between sub-structures. } \\
\text { (-) Difficult to see variable usage and branching conditions. } \\
\text { (-) Difficult to see how many endings there are. }\end{array}$ \\
\hline \multicolumn{2}{|c|}{ Player Interaction } \\
\hline $\begin{array}{l}\text { Capture when and how feedback is given. } \\
\text { Show when there is no feedback for an action. } \\
\text { Clarify when a particular branch will be visible to player or hidden. } \\
\text { Don't expect "click to continue" to be treated as an interaction. }\end{array}$ & $\begin{array}{l}\text { (+) Differentiates interaction styles: dialogue choices, interface } \\
\text { interactions, etc. } \\
\text { (+) Shows the possibility space of actions. } \\
\text { (+) Clearly differentiates events and feedback. } \\
\text { (-) Depending on player's perspective, feedback and events may } \\
\text { be perceived differently, and representation does not show this. }\end{array}$ \\
\hline \multicolumn{2}{|c|}{ Content and Characters } \\
\hline $\begin{array}{l}\text { Capture how much dialogue is present. } \\
\text { Categorize dialogue: narration, monologue, conversation, etc. } \\
\text { Understand how dialogue reveals character relationships. } \\
\text { Analyze whether dialogue shows bias or stereotypes. } \\
\text { Understand the visual layout and setting, character positioning. }\end{array}$ & $\begin{array}{l}\text { (-) Does not separate "caption" of a dialogue choice from the } \\
\text { choice itself - it should be an event or feedback. } \\
\text { (-) Does not clearly differentiate types of dialogue, events, etc. (i.e. } \\
\text { animation vs. UI vs. dialogue) } \\
\text { (-) Representation performs little or no content analysis. }\end{array}$ \\
\hline \multicolumn{2}{|c|}{ Experiment Design and Style } \\
\hline $\begin{array}{l}\text { Show what types of choices or branches are being visualized: object } \\
\text { selection, dialogue choices, etc. } \\
\text { Understand framing and context, such as player perspective (main } \\
\text { character, omniscience, etc.). } \\
\text { Show when part of the scenario is a framing narrative or tutorial. }\end{array}$ & $\begin{array}{l}\text { (-) Does not capture intent of designer's presentation and style } \\
\text { decisions. } \\
\text { (-) Different stylistic/implementation methods for choices lead to } \\
\text { different representations. } \\
\text { (-) Large scenarios may contain many nodes, and combining similar } \\
\text { ones could reduce confusion. }\end{array}$ \\
\hline
\end{tabular}

Table 2: Themes from the experts' expectations for the representation, and from their reviews of the current representation. Positive themes in the review section are noted with (+), and negative themes are noted with a (-).

\subsection{Representational Successes}

The algorithm described in 3.4 is a new model for static analysis of interactive narrative capable of transforming narrative scenarios into a visual and structured representation. It was used in previous work to build a set of metrics [34] for StudyCrafter scenarios, however it is generalizable to other contexts. For instance, we have manually analyzed other interactive narratives using the Interaction Map framework that underlies parts of this representation. With modifications to incorporate particular interaction affordances of other game design tools, a similar algorithm could automatically derive representations for scenarios built in those tools.

The evaluation walkthroughs highlight several ways in which the representation reveals and clarifies aspects of the scenario designs that our experts consider important. The graph-based structure of the representation enabled our experts to visually comprehend several relevant features of the scenarios: their linearity and branching structure, the relative lengths of branches, and the possibility spaces of interaction. Whereas the script editor built into StudyCrafter felt disorganized and overly complex for this analysis, the representation alleviated the confusion.

The experts found the clarity of the interaction maps' annotations of which nodes represented events versus feedback for player actions to be relevant and helpful. This visual depiction of the flow of particular actions and dialogue could enable designers to track whether, and to what extent, players would receive specific feedback for their choices. Had the designers who built these two scenarios visualized their linearity, their limited player interaction, and the lack of meaningful feedback for some of those interactions, they might have been able to use those insights to ameliorate some of the design choices that led to negative comments from our experts.

\subsection{Elaborating and Clarifying the Representation}

Though the representation fulfilled many of the experts' desires and clarified several aspects of the scenario designs, the experts also described several potential improvements, and they commented on several points of confusion. These included a lack of detail about branches and variable usage, the need for further clarification of perceived events versus feedback, a desire for additional annotations of specific sub-types and perceptual attributes of nodes, and a request for collapsing or merging of nodes to reduce clutter.

In the interaction map, we chose to depict only visible interactions with the scenario, theorizing that the designer would want to see only the parts of the scenario that were directly perceivable by the player. Experts, however, found the resulting connections between nodes, as shown in Figure 2 (c), confusing and difficult to interpret. Because we did not include branches as separate nodes, 
it looked like players could transition back to previous nodes when, in fact, the scenario was simply waiting for them to continue.

This particular interaction also appeared to allow any branch to be reached from any button. Only one transition would actually occur, depending on the button pressed, but we did not analyze the variable usage to show this. Visualizing variables and conditions, along with a separate branch node, would resolve this issue and enable designers to understand the actual flow of their scenario.

This ties into the more general need the experts expressed to better understand dependencies in the scenario. Many choice-based narrative games, and narrative-heavy games more broadly, include puzzles or gating on prior actions to unlock future interaction options. Thus, we see a general need for dependency visualization.

The experts also requested additional annotations in other cases. In the interaction maps, we visualized the overall types of nodes (interaction points, events, feedback), but we did not fully explain the ways in which those nodes would be presented to the player. Was an interaction point a dialogue choice or a button press? Was a feedback node an animation, a sound, or a dialogue? The experts would need to refer to the script graph to find out, and the connections between the graphs were not always easily determined. We could annotate these categories on the interaction map.

The experts also felt that some nodes, such as repetitive visibilitychange actions that had little effect on player experience, were redundant and unimportant. We could combine or collapse some of the nodes that are merely linear segments of events or feedback, replacing them with compact annotations. Alternatively, we could build a dynamic viewer for the representation that allows expanding and collapsing sections as designers work at various levels of granularity. This might also help us to build graphs of the entire scenario, combining all scenes, as requested by our experts.

A more dynamic set of views could also be beneficial in visualizing differing player perspectives. We could annotate certain nodes as feedback in some such perspectives, based on the player's knowledge of the consequences of choices gained by repeated playthroughs. In a first-playthrough perspective, however, they could be marked as events due to lack of knowledge of the narrative's scope. This is feasible because the representation is already designed to support structural analysis of interaction and its effects.

Finally, the experts suggested several improvements that are significantly out of scope for our current representation. These are primarily related to deep analysis of the content and meaning of a narrative, such as analysis of the perspectives and viewpoints of players, the relationships between various characters, and between a player and those characters, the use of biased language and stereotypes, and other questions of culturally-informed meaning.

\section{CONCLUSION}

We have described our procedure for constructing a theory-driven representation to model narrative-driven games, as operationalized for the StudyCrafter platform. We have presented an initial evaluation of this representation, in which experts on our research team used a cognitive walkthrough method to perform a detailed examination of two interactive narrative projects. The evaluation indicated that our representation was successful in illuminating the structures and interactive elements of these scenarios. On the other hand, it also revealed significant shortcomings and points of confusion. The evaluation itself was limited in scope, in that it only included members of our research team.

The insights from this expert evaluation can be useful to other game designers, researchers, or critics. The cognitive walkthroughs evinced many critiques of the scenarios' narrative structures: the overuse of framing narrative, the linearity and lack of interaction opportunities, and the feelings of constrained agency that resulted from these choices. These areas of focus and critique, and the representations and procedures that enabled them, may be relevant to many other games and interactive experiences.

By visualizing the narrative structure in graph form, we empowered experts to develop critiques with reference to specific points in the scenario design. They interrogated particular design decisions, such as the use of complex branching structures to simulate simple dialogue choices. Combining the representation's visual structure with the cognitive walkthrough method ensured that each moment of the scenario could be fully explored and described with clear, common reference points. A melding of theory-driven visualization and careful design review can thus be a valuable combination for work on interactive experiences.

In limiting the evaluation to members of the research team, however, this evaluation is necessarily constrained by our own perspectives, biases, and goals. Several members of the research team who acted as experts were also involved in the development of the representation, which influenced their comments. Future studies should incorporate designers from varied backgrounds, with various goals, to evaluate the representation in varied contexts.

Additionally, the scenarios in this study were built by designers who were not involved as experts. It would be useful to study designers' impressions of the representation when analyzing their own work, and how those impressions differ from those of external experts who did not create the scenario.

We plan to iterate on and improve the representation in response to the experts' feedback. First, we will update the Interaction Map framework to incorporate additional node types, annotations, and levels of detail to respond to the experts' feedback. Then, we will build a new computational implementation and visualization that incorporates this new theory and better supports designers. We plan to test this improved version of the representation in the context of designers' real workflows.

These representation efforts are building towards an AI-assisted creativity support tool that incorporates the representation as a sort of "knowledge base," much as other game AI agents use a perception and world representation system. We believe that these insights about how experts use our representation to evaluate interactive narrative scenarios will be essential in building automated tools that prompt reflection, provide feedback, or generate suggestions for narrative-focused games.

\section{ACKNOWLEDGMENTS}

This work was made possible by a grant from the NSF (IIS-1736185). Other work on StudyCrafter has also been supported by DARPA and Northeastern University. Thanks to the entire StudyCrafter team for making this work possible, and especially to Dylan Schouten for leading important organizational efforts. 


\section{REFERENCES}

[1] Manuel Araújo and Licinio Roque. 2009. Modeling Games with Petri Nets. Breaking New Ground: Innovation in Games, Play, Practice and Theory - Proceedings of DiGRA 2009 (2009)

[2] Sam Kabo Ashwell. 2015. Standard Patterns in Choice-Based Games. https://heterogenoustasks.wordpress.com/2015/01/26 standard-patterns-in-choice-based-games/

[3] Sander Bakkes and Joris Dormans. 2010. Involving player experience in dynamically generated missions and game spaces. In Eleventh International Conference on Intelligent Games and Simulation (Game-On'2010). 72-79.

[4] Mark Bernstein. 1998. Patterns of Hypertext. In Proceedings of the Ninth ACM Conference on Hypertext and Hypermedia: Links, Objects, Time and Space (HYPERTEXT '98). ACM, New York, NY, USA, 21-29. https://doi.org/10.1145/276627.276630

[5] Daniel Bormann and Tobias Greitemeyer. 2015. Immersed in Virtual Worlds and Minds: Effects of In-Game Storytelling on Immersion, Need Satisfaction, and Affective Theory of Mind. Social Psychological and Personality Science 6, 6 (Aug. 2015), 646-652. https://doi.org/10.1177/1948550615578177

[6] Emily Brown and Paul Cairns. 2004. A grounded investigation of game immersion. ACM Press, 1297. https://doi.org/10.1145/985921.986048

[7] Rogelio E Cardona-Rivera, Justus Robertson, Stephen G Ware, Brent Harrison, David L Roberts, and R Michael Young. 2014. Foreseeing Meaningful Choices. In Proceedings of the Tenth Annual AAAI Conference on Artificial Intelligence and Interactive Digital Entertainment (AIIDE 2014). AAAI, 7

[8] Elin Carstensdottir, Erica Kleinman, and Magy Seif El-Nasr. 2017. Towards an Interaction Model for Interactive Narratives. ICIDS 2017 (2017)

[9] Elin Carstensdottir and Magy Seif El-Nasr. 2018. Interaction Maps for Interactive Narratives. Technical Report NU-CCIS-TR-2018-001. College of Computer and Information Science, Northeastern University, Boston, MA.

[10] Joaquim Colás, Alan Tapscott, Valeria Righi, Ayman Moghnieh, and Josep Blat. 2017. Interaction and Outcomes in Collaborative Storytelling Systems: a Framework, a Field Study, and a Model. Computer Supported Cooperative Work (CSCW) 26, 4-6 (Dec. 2017), 627-662. https://doi.org/10.1007/s10606-017-9290-0

[11] Timothy Day and Jichen Zhu. 2017. Agency Informing Techniques: Communicating Player Agency in Interactive Narratives. In Proceedings of the 12th International Conference on the Foundations of Digital Games (FDG '17). ACM, New York, NY, USA, 56:1-56:4. https://doi.org/10.1145/3102071.3106363

[12] Joris Dormans. 2011. Simulating mechanics to study emergence in games. In Workshops at the Seventh Artificial Intelligence and Interactive Digital Entertainment Conference.

[13] Yellowlees Douglas and Andrew Hargadon. 2000. The Pleasure Principle: Immersion, Engagement, Flow. In Proceedings of the Eleventh ACM on Hypertext and Hypermedia (HYPERTEXT '00). ACM, New York, NY, USA, 153-160. https://doi.org/10.1145/336296.336354

[14] Steven P. Dow. 2008. Understanding user engagement in immersive and interactive stories. Ph.D. Dissertation. Georgia Institute of Technology.

[15] Matthew William Fendt, Brent Harrison, Stephen G. Ware, Rogelio E. CardonaRivera, and David L. Roberts. 2012. Achieving the Illusion of Agency. In Interactive Storytelling (Lecture Notes in Computer Science). Springer, Berlin, Heidelberg, 114125. https://doi.org/10.1007/978-3-642-34851-8 11

[16] Christian Guckelsberger, Christoph Salge, Jeremy Gow, and Paul Cairns. 2017. Predicting Player Experience Without the Player.: An Exploratory Study. In Annual Symposium on Computer-Human Interaction in Play (CHI PLAY '17). ACM, New York, NY, USA, 305-315. https://doi.org/10.1145/3116595.3116631

[17] Matthew Guzdial and Mark Riedl. 2018. Automated Game Design via Conceptual Expansion. In Fourteenth Artificial Intelligence and Interactive Digital Entertainment Conference.

[18] Casper Harteveld, Amy Stahl, Gillian Smith, Cigdem Talgar, and Steven C. Sutherland. 2016. Standing on the Shoulders of Citizens: Exploring Gameful Collaboration for Creating Social Experiments. In 49th Hawaii International Conference on System Sciences. 74-83. https://doi.org/10.1109/HICSS.2016.18

[19] C. Holmgard, M. C. Green, A. Liapis, and J. Togelius. 2018. Automated Playtesting with Procedural Personas with Evolved Heuristics. IEEE Transactions on Games (2018), 1-1. https://doi.org/10.1109/TG.2018.2808198

[20] Aaron Isaksen, Daniel Gopstein, and Andrew Nealen. 2015. Exploring Game Space Using Survival Analysis.. In Proceedings of the 10th International Conference on the Foundations of Digital Games (FDG 2015). ACM, Pacific Grove, CA, USA.

[21] Oleksandra Keehl and Adam M Smith. 2018. Monster Carlo: an MCTS-based Framework for Machine Playtesting Unity Games. In 2018 IEEE Conference on Computational Intelligence and Games (CIG). IEEE, 8.

[22] Brenda Laurel. 1986. Toward the design of a computer-based interactive fantasy system. $\mathrm{PhD}$ Thesis. The Ohio State University.

[23] Young-Seol Lee and Sung-Bae Cho. 2011. Context-Aware Petri Net for Dynamic Procedural Content Generation in Role-Playing Game. Computational Intelligence Magazine, IEEE 6 (June 2011), 16-25. https://doi.org/10.1109/MCI.2011.940618

[24] Craig A. Lindley. 2005. Story and narrative structures in computer games. In Developing Interactive Narrative Content, Brunhild Bushoff (Ed.). HighText Verlag, Munich.
[25] Thomas Mahatody, Mouldi Sagar, and Christophe Kolski. 2010. State of the Art on the Cognitive Walkthrough Method, Its Variants and Evolutions. International fournal of Human-Computer Interaction 26, 8 (July 2010), 741-785. https://doi. org $/ 10.1080 / 10447311003781409$

[26] Chris Martens and Matthew A. Hammer. 2017. Languages of Play: Towards Semantic Foundations for Game Interfaces. In Proceedings of the 12th International Conference on the Foundations of Digital Games (FDG '17). ACM, New York, NY, USA, 32:1-32:10. https://doi.org/10.1145/3102071.3102096

[27] Michael Mateas. 2002. Interactive Drama, Art and Artificial Intelligence. $\mathrm{PhD}$ Thesis. Carnegie Mellon University, Pittsburgh, PA, USA.

[28] Michael Mateas and Noah Wardrip-Fruin. 2009. Defining Operational Logics. In Proceedings of DiGRA 2009.

[29] Alison McMahan. 2003. Immersion, engagement and presence. The video game theory reader 67 (2003), 86.

[30] David E. Millard, Charlie Hargood, Michael O. Jewell, and Mark J. Weal. 2013. Canyons, deltas and plains: towards a unified sculptural model of location-based hypertext. In 24th ACM Conference on Hypertext and Social Media. ACM, 109-118.

[31] Brian Moriarty. 2015. I Sing the Story Electric. http://ludix.com/moriarty/ electric.html

[32] Janet Horowitz Murray. 1997. Hamlet on the Holodeck: The Future of Narrative in Cyberspace. The Free Press, New York, NY, USA.

[33] Joseph C. Osborn. 2017. Operationalizing Operational Logics: Semiotic Knowledge Representations for Interactive Systems. In Proceedings of the TwentySixth International foint Conference on Artificial Intelligence, IfCAI-17. 5199-5200. https://doi.org/10.24963/ijcai.2017/759

[34] Nathan Partlan, Elin Carstensdottir, Sam Snodgrass, Erica Kleinman, Gillian Smith, Casper Harteveld, and Magy Seif El-Nasr. 2018. Exploratory Automated Analysis of Structural Features of Interactive Narrative. In Proceedings of the 14th AAAI Conference on Artificial Intelligence and Interactive Digital Entertainment. The AAAI Press, Palo Alto, California, Edmonton, AB, Canada.

[35] Christian Roth and Hartmut Koenitz. 2017. Towards Creating a Body of Evidence-based Interactive Digital Narrative Design Knowledge: Approaches and Challenges. In Proceedings of the 2Nd International Workshop on Multimedia Alternate Realities (AltMM '17). ACM, New York, NY, USA, 19-24. https: //doi.org/10.1145/3132361.3133942

[36] Christian Roth, Peter Vorderer, and Christoph Klimmt. 2009. The Motivational Appeal of Interactive Storytelling: Towards a Dimensional Model of the User Experience. In Interactive Storytelling (Lecture Notes in Computer Science). Springer, Berlin, Heidelberg, 38-43. https://doi.org/10.1007/978-3-642-10643-9_7

[37] James Ryan. 2017. Grimes' Fairy Tales: A 1960s Story Generator. In 10th International Conference on Interactive Digital Storytelling (Lecture Notes in Computer Science), Nuno Nunes, Ian Oakley, and Valentina Nisi (Eds.), Vol. 10690. Springer, Cham, Funchal, Madeira, Portugal. https://doi.org/10.1007/978-3-319-71027-3_8

[38] Tom Schaul. 2013. A video game description language for model-based or interactive learning. In IEEE Conference on Computational Inteligence in Games (CIG). IEEE, 1-8.

[39] Henrik Schoenau-Fog. 2011. Hooked! - Evaluating Engagement as Continuation Desire in Interactive Narratives. In Interactive Storytelling (Lecture Notes in Computer Science). Springer, Berlin, Heidelberg, 219-230. https://doi.org/10.1007/ 978-3-642-25289-1_24

[40] Magy Seif El-Nasr. 2007. Interaction, narrative, and drama: Creating an adaptive interactive narrative using performance arts theories. Interaction Studies 8 (June 2007), 209-240. https://doi.org/10.1075/is.8.2.03eln

[41] Emily Short. 2016. Small-Scale Structures in CYOA. https://emshort.blog/2016/ $11 / 05 /$ small-scale-structures-in-cyoa/

[42] Rick Spencer. 2000. The streamlined cognitive walkthrough method, working around social constraints encountered in a software development company. In Proceedings of the SIGCHI conference on Human Factors in Computing Systems. ACM, 353-359.

[43] Adam Summerville, Chris Martens, Ben Samuel, Joseph Osborn, Noah WardripFruin, and Michael Mateas. 2018. Gemini: Bidirectional Generation and Analysis of Games via ASP. In AIIDE 2018. 7.

[44] Nicolas Szilas and Ioana Ilea. 2014. Objective Metrics for Interactive Narrative. In Interactive Storytelling (Lecture Notes in Computer Science). Springer, Cham, 91-102. https://doi.org/10.1007/978-3-319-12337-0_9

[45] Karen Tanenbaum and Theresa Jean Tanenbaum. 2010. Agency as commitment to meaning: communicative competence in games. Digital Creativity 21, 1 (March 2010), 11-17. https:// doi.org/10.1080/14626261003654509

[46] David Thue, Vadim Bulitko, Marcia Spetch, and Trevon Romanuik. 2011. A Computational Model of Perceived Agency in Video Games.. In Proceedings of the Seventh AAAI Conference on Artificial Intelligence and Interactive Digital Entertainment. Stanford, California, USA.

[47] Ivar E. Vermeulen, Christian Roth, Peter Vorderer, and Christoph Klimmt. 2010. Measuring User Responses to Interactive Stories: Towards a Standardized Assess-ment Tool. In Interactive Storytelling (Lecture Notes in Computer Science). Springer, Berlin, Heidelberg, 38-43. https:// doi.org/10.1007/978-3-642-16638-9 7

[48] Noah Wardrip-Fruin, Michael Mateas, Steven Dow, and Serdar Sali. 2009. Agency Reconsidered. In Proceedings of DiGRA 2009. 